\title{
Osteoporose-Prophylaxe: Wissenschaftliche Methoden belegen die Bedeutung der Ernährung
}

\author{
Peter Burckhardt \\ Clinique Bois-Cerf SA, CH-Lausanne
}

nfolge von Osteoporose auftretende Knochenfrakturen werden meist als Schicksalsschläge hingenommen, weil sie genetisch determiniert und mit dem normalen Alterungsprozess assoziiert sind. Sie können jedoch zum Teil verhindert werden. An erster Stelle steht dabei die medikamentöse Therapie. Allerdings sollte auch die Prophylaxe nicht ausser Acht gelassen werden, da sie - lebenslang angewendet - eindeutig positive Wirkungen zeigt. Dabei kommt der Ernährung eine besondere Bedeutung $\mathrm{zu}$, neben körperlichem Training im Wachstumsalter und der Sturzprophylaxe im höheren Alter. Die vorhandenen wissenschaftlichen Grundlagen sollen im Folgenden diskutiert werden.

\section{Methoden}

Diätetische Ratschläge sind populär. Oft fehlt ihnen aber die wissenschaftliche Basis und somit ihre Berechtigung. Jedes Gerücht, jeder Erfahrungsbericht, jede Beobachtung beim Tier wird eilig als Grundlage für neue Theorien und Ratschläge aufgegriffen. Der Einfluss der Ernährung auf die Knochengesundheit lässt sich jedoch wissenschaftlich untersuchen. Dabei muss stets eine der vielen Methoden zur Erfassung der Nahrungseinnahme eingesetzt werden, wie spezifische Fragebögen, Tagebücher, Interviews, Kontrollen. Vor- und Nachteile der verschiedenen Methoden werden hier nicht diskutiert. Auf der anderen Seite
Hintergrund: Neue Forschungsergebnisse belegen die Bedeutung der Ernährung bei der Osteoporose-Prävention, d.h. im Hinblick auf die Erhaltung der Knochensubstanz bei älteren und die Stimulierung des Knochenwachstums bei jungen Menschen. Die vorliegenden Studienergebnisse sind nicht immer schlüssig, da jede Hypothese zuerst in Querschnitts-Studien geprüft, dann mit Verlaufs-Studien erhärtet, und schliesslich, wenn möglich, in Interventions-Studien bewiesen wird. Zielsetzung: Zusammenfassung und Kommentierung wissenschaftlich belegter Fakten zur Ernährungsforschung bei Osteoporose Methoden: Auswertung der Literatur, hauptsächlich aus den Jahren 2000-2005, aus den gängigen elektronischen Datenbanken. Ergebnisse: Der positive Effekt von Calcium und Vitamin D ist erwiesen, ebenso der negative Einfluss eines Proteinmangels, wobei die positive Wirkung von Protein auf die Calcium-Absorption und auf die Sekretion des Wachstumshormons IGF untersucht werden, sowie die vielleicht spezifische Wirkung bestimmter Aminosäuren. Ebenfalls wird zur Zeit die Bedeutung von Kalium, Vitamin K und anderen Substanzen untersucht. Schliesslich wurde auch die positive Wirkung von Milchprodukten sowie von Früchten und Gemüsen aufgezeigt. Dabei scheint eine basische Ernährung eine entscheidende Rolle zu spielen. Schlussfolgerung: Die Ernährung beeinflusst die Knochengesundheit während des ganzen Lebens, wobei nicht nur Calcium und Vitamin D, sondern auch Vitamin K, Kalium, bestimmte Aminosäuren, weitere Vitamine sowie auch eine basenliefernde Nahrung anscheinend eine positive Wirkung haben.

Schlüsselwörter: Osteoporose, Ernährung, Vitamine, Proteine, Calcium, Säure-Basen

\section{Prevention of Osteoporosis:}

\section{Scientific Evidence Supports the Impact of Nutrition on Bone Health}

Background: New research provides evidence for the role of nutrition in the prevention of osteoporosis, i.e. for maintenance of bone mass in the elderly and for the stimulation of bone development in the younger. However, the studies are of inconsistent significance since each hypothesis is first tested by cross-sectional studies, then evaluated by longitudinal studies, and finally tested - whenever possible - in intervention trials. Objective: To give an overview of the evidence concerning nutritional research in osteoporosis. Methods: Analysis of the recent literature on osteoporosis and nutritional research from main data bases, mainly from 2000 to 2005. Results: The positive role of calcium and vitamin D for bone health is established, the negative impact of protein deficiency too, while the positive effect of protein on calcium absorption and the secretion of the growth hormone IGF is under examination, as well as the specific action of some aminoacids. In addition, the positive role of Potassium, vitamin K, and other substances are under investigation. Finally, the positive impact of dairy products, as well as of fruits and vegetables can also be demonstrated. In this context, the alkali load seems to be crucial. Conclusion: Nutrition influences bone health throughout life. Not only calcium and vitamin $D$, but also vitamin $K$, potassium, certain amino acids, other vitamins and the nutritional alkali load seem to play a positive role.

Key words: osteoporosis, nutrition, vitamins, proteins, calcium, acid-base 
Tab. 1. Wissenschaftlich nachgewiesene nahrungsbedingte Einflüsse auf die Knochengesundheit (hauptsächlich auf die Knochendichte)

\begin{tabular}{|c|c|c|c|}
\hline Nahrungsfaktor & $\begin{array}{l}\text { Grössere } \\
\text { Querschnitts } \\
\text {-studien }\end{array}$ & $\begin{array}{l}\text { Verlaufs- } \\
\text {-studien }\end{array}$ & $\begin{array}{l}\text { Interventions- } \\
\text {-studien }\end{array}$ \\
\hline Calcium & $K, E, A$ & $\mathrm{~K}, \mathrm{~A}$ & $K, E^{*}, A^{*}$ \\
\hline Phosphor & - & - & (A) \\
\hline Protein & E & $E^{*}$ & A (mit Calcium) \\
\hline Kalium & $\mathrm{E}$ & mit Magnesium: E & - \\
\hline Magnesium & - & mit Kalium: E & - \\
\hline Vitamin D & $E$ & - & $E, A^{*}$ \\
\hline Vitamin $\mathrm{K}$ & E & $E^{*}$ & $E(*)$ \\
\hline Vitamin C & - & $\mathrm{E}$ & - \\
\hline Bicarbonat & - & - & $E$ \\
\hline Milchprodukte & $\mathrm{K}, \mathrm{E}$ & $\mathrm{K}$ & $\mathrm{K}, \mathrm{E}$ \\
\hline Früchte, Gemüse & $E, A$ & $\mathrm{~K}, \mathrm{E}$ & - \\
\hline Alkalis. Nahrung & $E, A$ & - & - \\
\hline Fleisch & $E, A$ & $E$ & - \\
\hline Soya & $E ?$ & - & $\mathrm{E}$ ? \\
\hline
\end{tabular}

( $K=$ Kinder, $E=$ Erwachsene, $A=$ spezifisch nur Ältere,

* auch Anti-Fraktur-Wirkung prospektiv nachgewiesen)

wird die Knochengesundheit bewertet, indem die Knochendichte gemessen oder Frakturen erfasst werden.

Als erstes sind die Querschnittsstudien zu erwähnen. Dabei werden in einer definierten Bevölkerungs- und Altersgruppe gleichzeitig die Knochendichte und die Einnahme eines bestimmten Nahrungsmittels (z.B. Milchprodukte) oder einer Nahrungskomponente (z.B. Calcium, Vitamin K) gemessen und auf ihre Korrelation hin geprüft. Dabei müssen stets andere Einflüsse, wie Lebensgewohnheiten, mit berücksichtigt werden. So fand man z.B. heraus, dass die Einnahme von Calcium im Wachstumsalter und im höheren Alter wichtig ist, weniger aber beim jungen Erwachsenen.

Die zweite und bessere Untersuchungsmethode sind Verlaufsstudien. Dabei wird der Verlauf der Knochengesundheit über einen bestimmten Zeitraum, meistens 2 bis 3 Jahre, mit der Einnahme eines bestimmten Nahrungsmittels oder bestimmter Nahrungsmittelkomponenten verglichen. Bei Jugendlichen wird das Knochenwachstum erfasst, bei Älteren der Knochenverlust oder das Auftreten von Frakturen.

Die dritte und aufschlussreichtse Methode ist die Interventionsstudie, bei der ein Nahrungsmittel über eine bestimmte Zeit verabreicht wird, und die Entwicklung der Knochendichte oder die Anzahl auftretender Frakturen verglichen werden mit den entsprechenden Daten einer Kontrollgruppe, bei der die Ernährung nicht unverändert bleibt. Eine derartige Studie kann nur mit bestimmten Nahrungsmitteln (z.B. Milch) oder gar nur mit einem Supplement (z.B. Calcium, Vitamin D, Soya) durchgeführt werden. Es ist nicht möglich, den Versuchspersonen über längere Zeit ganze Ernährungsmuster aufzuzwingen.

Tabelle 1 zeigt, welche positiven Wirkungen der Ernährung auf den Knochen bis jetzt nachgewiesen worden sind.

\section{Der Einfluss von Nahrungskomponenten}

\section{Calcium}

Calcium ist die meist untersuchte Nahrungskomponente. In zahlreichen Querschnitts-, Verlaufs- und Interventionsstudien ist gezeigt worden, dass Calcium das Knochenwachstum fördert [1] und im Alter den Knochenabbau verlangsamt [2]. Ist die Evaluie- rungsmethode aber oberflächlich, können falsch negative Resultate produziert werden. Zum Beispiel kann die Angabe über den Milchkonsum nicht als Kriterium für die gesamte Calciumzufuhr angesehen werden [3].

Beim Erwachsenen im mittleren Alter ist der Nachweis allerdings nicht immer gelungen, ausser in Bevölkerungsgruppen mit besonders tiefer Calciumeinnahme, wie z.B. Chinesen. Damit ein Calciummangel als Ursache von Osteoporose auftritt, muss die Einnahme sehr gering sein oder es müssen Probleme bei der Absorption vorliegen.

\section{Vitamin D}

Aus mehreren Querschnittsstudien ist bekannt, dass eine deutliche Beziehung besteht zwischen den Blutspiegeln von Vitamin D und der Knochendichte, der Sturzgefahr und der Frakturhäufigkeit. Vitamin D ist wohl in Fischöl reichlich vorhanden, und ein wenig auch in Milchprodukten, aber es wird vorwiegend vom Körper selber hergestellt, sofern die Haut eine ausreichende Sonnenbestrahlung erhält. Dies ist aber häufig nicht der Fall. Querschnittsstudien haben gezeigt, dass die Blutspiegel von Vitamin D bei fast allen Altersheiminsassen zu niedrig sind, oft auch bei älteren Personen, und auch bei vielen jungen Menschen. Interventionsstudien konnten dann nachweisen, dass eine Substitution mit Vitamin D und Calcium bei Älteren, vor allem bei Altersheiminsassen, das Auftreten von Frakturen teilweise verhindert [4]. Die optimale Dosis liegt bei 800 - 1000 IE pro Tag [5]. Zudem verringert Vitamin D auch die Sturzgefahr [6]. Aus diesen Gründen sind Calcium und Vitamin D Bestandteile jeder Osteoporose-Prophylaxe und ergänzen immer eine medikamentöse Therapie der Osteoporose.

\section{Proteine}

Dass eine extrem proteinreiche Ernährung knochenschädlich ist, konnte beim Menschen aus ethischen Gründen nicht nachgewiesen werden. Aber dass eine proteinarme Ernährung schädlich ist, ist eindeutig belegt [7] Verlaufsstudien haben gezeigt, dass eine proteinarme Ernährung mit be- 
schleunigtem Knochenverlust und erhöhter Frakturhäufigkeit einhergeht [8, 9]. Die grossen Querschnittsstudien, welche die Protein-Einnahme mit der Knochendichte vergleichen, sind allerdings nicht immer schlüssig. Ein Grund dafür liegt darin, dass die positive Wirkung der Proteine nur dann zum Zuge kommt, wenn auch eine ausreichende Einnahme von Calcium und Vitamin D gewährleistet ist [10]. Auf der anderen Seite kann der negative Einfluss einer proteinarmen Ernährung am besten bei Personen nachgewiesen werden, deren CalciumEinnahme gering ist [11]. Analog gilt, dass eine Calcium-Substitution am Schenkelhals nur dann voll wirksam ist, wenn die Protein-Einnahme hoch genug ist [12].

Die Art der Proteine scheint eine gewisse Rolle $\mathrm{zu}$ spielen. Tierische Proteine, d.h. Fleisch, scheinen den Knochenabbau zu beschleunigen, während pflanzliche Proteine den Knochen erhalten [13]. Dies ist noch nicht eindeutig bestätigt worden. Die unterschiedliche Säurebelastung dieser Nahrungsproteine mag hier den Ausschlag geben (siehe unten). Schädlicher aber als eine ungünstige Natur der Proteine ist eine proteinarme Ernährung, wie sie bei vielen älteren Personen $\mathrm{zu}$ finden ist. Werden schlecht ernährten älteren Personen proteinhaltige Supplemente verabreicht, so steigt die Knochendichte an oder sinkt weniger schnell ab [14]. Dies hängt wohl nicht mehr so sehr von der Natur der Proteine ab. Ein Teil der Wirkung der Proteine kann dem Anstieg des Wachstumshormons IGF zugeschrieben werden.

\section{Vitamin K}

Vitamin K wirkt als Co-Enzym bei der Carboxylierung von Osteocalcin, einem Protein des Grundgerüstes der Knochen, welches letzterem grössere Festigkeit verleiht. Vitamin-K-Mangel korreliert daher vermehrt mit unreifem Osteocalcin, tieferer Knochendichte und mit einer grösseren Anzahl von Frakturen. Querschnittsstudien haben dies deutlich gezeigt, obwohl einige Studien nur bei Frauen durchgeführt wurden [15]. Eine Verlaufsstudie zeigte auch eine Vitamin-K- abhängige Frakturrate, allerdings erst nach dem 5. Beobachtungsjahr [16]. Eine grössere Interventionsstudie mit Vitamin $\mathrm{K}$ ist zur Zeit im Gang. Es müssen jedoch zuerst ihre Resultate abgewartet werden, bevor eine Empfehlung für Vitamin $\mathrm{K}$ ausgesprochen werden kann. Das würde auch dem derzeitigen Trend zum vermehrten Verzehr von Früchten und Gemüse (siehe unten) Auftrieb verleihen.

\section{Vitamin A}

Die Zufuhr von Vitamin A in grösseren Mengen, wie dies durch übermässigen Verzehr von Fischölen möglich ist, hat eine negative Auswirkung auf die Knochengesundheit [17, 18, 19].

\section{Vitamin C}

Einige wenige Studien haben eine positive Wirkung von Vitamin C auf die Knochendichte von postmenopausalen Frauen gezeigt. Aber sie reichen nicht aus, um eine Vitamin-C-Supplementierung für die Prophylaxe der Osteoporose zu empfehlen [20].

\section{Magnesium}

Lediglich in Tierstudien, vor allem an Ratten, konnte dem Magnesium eine knochenschützende Wirkung zugeschrieben werden. Durch die Verfeinerung der diätetischen Erhebungen beim Menschen könnte sich die Möglichkeit ergeben, eine solche Beziehung zumindest in Querschnittsstudien aufzudecken.

\section{Kalium}

Kalium vermindert die Ausscheidung von Calcium über die Niere und verbessert die Calciumbilanz. Dies scheint ein Nieren-bezogener Effekt zu sein. Kalium-Bicarbonat sowie KaliumCitrat haben eine positive Wirkung auf den Knochstoffwechsel [21, 22]. Dabei ist aber noch nicht geklärt, ob dies dem Bicarbonat und dem Citrat zugeschrieben werden muss, da diese Substanzen die Säurebelastung vermindern (siehe unten).

\section{Der Einfluss von Nahrungsmitteln}

\section{Milchprodukte}

Milchprodukte sind Lieferanten von Calcium, Protein und Phosphor, und somit knochenfreundlich. In der Tat haben zahlreiche Querschnitts-, Verlaufs- und Interventionsstudien gezeigt, dass Milch und Milchprodukte positive Wirkungen auf die Knochendichte haben, und dies sowohl bei Kindern [23, 24], als auch bei älteren Personen [25]. Die Befürchtung, dass damit zu viel Cholesterin eingenommen würde, ist nicht berechtigt, zumal die fettarmen Milchprodukte nicht weniger Calcium enthalten und die fettarmen Käse calciumreich sind. Milchprodukte stellen zudem die beste Nahrungsquelle von Calcium dar, tragen aber nur ungefähr die Hälfte des Calciums aus der Ernährung bei. Und je genauer eine diätetische Erhebung ist, desto kleiner ist dieser Anteil, da auch Wasser, Mineralwasser, Gemüse und Früchte, Brot etc. als Calcium-Quellen mit einbezogen werden können.

\section{Mineralwasser}

Mineralwasser sind eine natürliche Quelle von Calcium, da sie oft mehr als 300 mg/l Calcium enthalten, vor allem die in der Schweiz verfügbaren. Einige wenige Studien zeigen, dass calciumreiche Mineralwasser den Knochenaufbau fördern, und dass der regelmässige Konsum von solchen Wassern mit einer höheren Knochendichte einhergeht [26]. Es gibt einige Hinweise, dass der Bicarbonat-Gehalt mindestens ebenso wichtig ist (siehe unten).

\section{Fleisch und Soya}

Dass ein hoher Fleischkonsum mit einer erhöhten Gefahr für Osteoporose und Frakturen einher geht, ist aus epidemiologischen Untersuchungen bekannt [27]. Vergleicht man aber die Proteineinnahme mit der Knochendichte oder dem Knochabbau, dann scheint hauptsächlich eine niedrige Proteineinnahme schädlich $\mathrm{zu}$ sein, wie bereits diskutiert. Es müsste jedoch zwischen Proteinen tierischer und pflanzlicher Herkunft unterschieden werden, wobei ein Überwiegen des tierischen Proteins gemäss einer 
Verlaufsstudie den Knochenabbau zu beschleunigen und das Frakturrisiko zu erhöhen scheint [13]. Von anderen Autoren ist dies noch nicht bestätigt worden, ausser bei einer kurzfristigen Interventionsstudie bei Bettlägerigen [28]. Soya als Proteinquelle könnte dabei von Interesse sein; Studien hierzu sind im Gang. Jedoch scheinen die in Soya vorhandenen Isoflavone wirkungslos zu sein [29].

\section{Früchte und Gemüse}

Der regelmässige Verzehr von Früchten und Gemüsen schützt den Knochen; dies ist eine neue Erkenntnis, die man aus grösseren Querschnitts- und Verlaufsstudien gewonnen hat [30, 31, 32]. Auch bei Jugendlichen wird der Knochenstoffwechsel dadurch günstig beeinflusst [33]. Es wird wohl schwierig sein, dieses Ergebnis mit Interventionsstudien zu untermauern. Tierversuche sind im Gang, um die knochenschützenden Komponenten von Früchten und Gemüse zu charakterisieren. Häufig wird auf die positive Wirkung von Kalium verwiesen, das ja hauptsächlich in Form von Früchten und Gemüse eingenommen wird. Dasselbe trifft auch für das Vitamin $\mathrm{K} \mathrm{zu}$. Vegetarier müssten somit über eine besonders gute Knochengesundheit verfügen. Diesbezügliche Querschnittsstudien zeigen wechselnde Resultate; im Allgemeinen haben Vegetarier eine normale Knochenmasse, wobei kein Unterschied zwischen Ovo-lacto-Vegetariern und Nicht-Vegetariern besteht [34]. Das erklärt sich durch die sehr unterschiedliche Protein- und Calciumzufuhr bei vegetarischen Ernährungsweisen.

\section{Die Theorie der Säurebelastung}

Die günstige Wirkung von Früchten und Gemüse, die möglicherweise negative Wirkung von erhöhtem Fleischkonsum, das Interesse am Bikarbonat im Mineralwasser, das alles sind Hinweise auf die wahrscheinliche Bedeutung der Säurebelastung.

Experimente an Knochenzellen, am Knochengewebe und am Tier haben gezeigt, dass der Knochen-aufbau und -abbau vom Säuregrad des Körperinneren abhängt. Nun ist dieser aber sehr genau geregelt. Die Regelung wird z.T. mittels des Knochengewebes bewerkstelligt. Der Knochen stellt ein grosses Reservoir von alkalisierenden Puffersubstanzen dar. Sobald der Organismus einer Säurebelastung ausgesetzt ist, werden die knochenbildenden Zellen gehemmt und die resorbierenden Zellen stimuliert, damit so dank erhöhter Knochenresorption mehr Bicarbonat zur Neutralisierung der Säuren freigesetzt wird. Wird andererseits Bicarbonat in reiner Form [21] oder mit der Nahrung [35] verabreicht, geht die Knochenresorption zurück, wie in kurzen Interventionsstudien gezeigt werden konnte.

Für jedes Nahrungsmittel lässt sich der Säuregehalt errechnen. Damit kann die mittlere Säurebelastung durch die Ernährung ermittelt werden. Sie steht in Korrelation mit der Säureausscheidung im Urin und mit der Ausscheidung von Calcium und von Abbauprodukten des Knochengewebes, der sogenannten Marker der Knochenresorption. Zur vereinfachten Schätzung des Säure-Potentials der Nahrung wird der NEAP (Net endogenous noncarbonic acid production) berechnnet aus dem Quotienten Proteine/Kalium, da die tierischen Proteine säurelastig sind, während Kalium sich in den meist basischen Früchten und Gemüse findet [36]. Es kann auch der PRAL (potential renal acid load) verwendet werden, welcher neben Protein und Kalium auch Phosphor, Magnesium und Calcium in die Rechnung einbezieht.

Mehrere Querschnittsstudien bei älteren Frauen haben deutliche Beziehungen nachgewiesen zwischen der so errechneten Säurebelastung durch die Nahrung und der Knochendichte einerseits, dem Knochabbau anderseits [32, 37], und auch der Frakturhäufigkeit. Aber dass eine Nahrungsumstellung auf eine basische Ernährung die Knochendichte verbessert, ist noch nicht nachgewiesen worden und wird aus praktischen Gründen auch schwierig nachzuweisen sein. Kurze Studien mit K-Citrat, mit K-Bikarbonat, mit alkalischer Nahrung und Mineralwasser haben zumindest deren güns- tige Beeinflussung des Knochenstoffwechsels zeigen können [21, 35, 38]. Im selben Zusammenhang könnte auch dem Soyaprotein, welches alkalisch ist, eine günstige Wirkung zugeschrieben werden, zumal es noch Isoflavon enthält. Es gibt interessante Hinweise [39], allerdings fehlen die Beweise.

Verlockend an dieser Theorie ist die Kongruenz mit den diätetischen Empfehlungen für die Verhütung von kardio-vaskulären Krankheiten, von Diabetes und von Adipositas, welche alle einen vermehrten Verzehr von Früchten und Gemüsen, und damit von Kalium und Bikarbonat, propagieren.

\section{Zusammenfassung}

Obschon eine genaue Erfassung der Nahrungsmitteleinnahme schwierig ist, hat die Forschung in den letzten 15 Jahren viele Erkenntnisse geliefert, die der Ernährung eine wichtige Rolle bei der Prävention der Osteoporose einräumen. Dies betrifft nicht nur den älteren Menschen, der seine Knochenmasse erhalten muss, sondern besonders auch das Kind und den Adoleszenten, der sein Skelett aufbaut und dann vom erworbenen Knochenkapital abhängt. Jede Hypothese wird zuerst mittels Querschnittsstudien geprüft, wo Knochendichte und Ernährung verglichen werden, dann mit Verlaufsstudien erhärtet, wo Knochenverlust und Frakturhäufigkeit erfasst werden, und schliesslich, wenn möglich, mit Interventionsstudien bewiesen. In diesem Zusammenhang ist die wesentliche Rolle von Calcium und Vitamin D erwiesen, die der Proteine erforscht, und diejenige von Kalium, Vitamin K und weiteren Substanzen wird zur Zeit untersucht. Schliesslich wurde auch die positive Wirkung von Milchprodukten sowie von Früchten und Gemüse nachgewiesen. Dabei scheint die Ernährung bei der Prävention der Säurebelastung eine signifikante Rolle zu spielen.

\section{Literatur}

1. Matkovic V, Goel PK, Badenhop-Stevens NE et al: Calcium supplementation and bone mineral density in females from childhood to young adulthood: a randomized controlled trial. Am J Clin Nutr 2005;81:175-188. 
2. Heaney RP: Calcium, diary products and osteoporosis. J. Am.Coll.Nutr. 2000;19 (2 suppl): $83 S$ - 99S

3. Kanis JA, Johansson $H$, Oden A, et al: A meta-analysis of milk intake and fracture risk: low utility for case finding. Ost Int. 2005; 16:799-804

4. Trivedi DP, Doll R, Khaw KT: Effect of four monthly oral vitamin D (cholecalciferol) supplementation on fractures and mortality in men and women living in the community: randomised double blind controlled trial. Brit Med J 2003;326:469-472.

5. Dawson-Hughes B: Estimates of optimal vitamin D status. Osteopororis Int 2005;16: 713-716.

6. Bischoff-Ferrari HA. Dawson-Hughes B. Willett WC. Staehelin HB. Bazemore MG. Zee RY. Wong JB. Effect of Vitamin D on falls: a meta-analysis. JAMA 2004;291:1999-2006.

7. Kerstetter JE, Looker AC, Insogna KL: Low dietary protein and low bone density. Calcif Tissue Int 2000;66(4):313.

8. Munger RG, Cerhan JR, Chiu BCH: Prospective study of dietary protein intake and risk of hip fracture in postmenopausal women. Am J Clin Nutr 1999;69:147-152.

9. Hannan MT, Tucker KL, Dawson-Hughes B et al: Effect of dietary protein on bone loss in elderly men and women: the Framingham Osteoporosis Study. J Bone Min Res 2000; 15:2504-2512.

10. Rapuri PB, Gallagher JC, Haynatzka V: Protein intake: effects on bone mineral density and the rate of bone loss in elderly women. Am J Clin Nutr 2003;77:1517-1525.

11. Hannan MT, Tucker KL, Dawson-Hughes B et al: Low calcium intake magnifies the bone loss seen with low dietary protein intake in elderly men and women. J BoneMin Res 2003;18 suppl. 2: S240.

12. Dawson-Hughes B, Harris SS: Calcium intake influences the association of protein intake with rates of bone loss in elderly men and women. Am J Clin Nutr 2002;75:773-779.

13. Sellmeyer DE, Stone KL, Sebastian A, et al: A high ratio of dietary animal to vegetable protein increases the rate of bone loss and the risk of fracture in postmenopausal women. Am J Clin Nutr 2001;73:118-122.

14. Schürch MA, Rizzoli R, Slosman D et al: Protein supplements increase serum insulin-like growth factur-I levels and attenuate proximal femur bone loss in patients with recent hip fractures. Ann Int Med 1998;128:801-809.

15. Booth SL, Broe KE, Gagnon DR, et al: Vitamin $\mathrm{K}$ intake and bone mineral density in women and men. Am J Clin Nutr 2003;77:512-516.

16. Booth SL, Tucker $\mathrm{KL}$, Chen $\mathrm{H}$, Hannan MT, Gagnon DR, Cupples A, Wilson PWF, Ordovas J, Schaefer EJ, Dawson-Hughes B Kiel DP. Dietary vitamin K intakes are associated with hip fracture but not with bone mineral density in elderly men and women. Am J Clin Nutr 2001;71:1201-1208.

17. Burckhardt $P$ : Vitamin $A$ and bone health. In Holick MF, Dawson-Hughes B (Hrsg): Nutrition and bone health. Humana Press, Totowa, NJ, US. 2004. S 391-402.

18. Melhus $H$, Michaëlsson $K$ Kindmark A Bergström R, Homberg L, Mallmin H, Wolk $A$, Ljunghall $S$. Excessive dietary intake of vit$\operatorname{amin} A$ is associated with reduced bone mineral density and increased risk for hip fracture. Ann Intern Med 1998;129:770-778.

19. Michaelsson K, Lithell H, Vessby B, Melhus $\mathrm{H}$ : Serum retinol levels and the risk of fracture. New Engl J Med 2003;348:287-294.

20. Hall SL, Greendale GA: The relation of dietary vitamin $\mathrm{C}$ intake to bone mineral density: results from the PEPI study. Calc Tissue Intern 1998;63(3):183-189.

21. Sebastian A, Harris ST, Ottaway JH, Todd KM, Morris RC: Improved mineral balance and skeletal metabolism in postmenopausal women treated with potassium bicarbonate. NEJM 1994;330(25):1776-1781.

22. Sakhaee K, Maalouf NM, Abrams SA, Pak CYC: Effects of potassium alkali and calcium supplementation on bone turnover in postmenopausal women. J Clin Endocrin Metab 2005;90:3528-3533.

23. Black RE, Williams SM, Jones IE, Goulding A: Children who avoid drinking cow milk have low dietary calcium and poor bone health. Am J Clin Nutr 2002;76:675-680.

24. Kalkwarf HJ, Khoury JC, Lamphear BP: Milk intake during childhood and adolescence, adult bone density and osteoporotic fractures in US women. Am J Clin Nutr 2003:77: 257-265.

25. McCabe LD, Martin BR, McCabe GP et al: Dairy intakes affect bone density in the elderly. Am J Clin Nutr 2004;80:1066-1074

26. Burckhardt P: Mineral waters : Effetcs on bone and bone metabolism. In: Burckhardt P, Dawson-Hughes P, Heaney RP (Hrsg): Nutritional aspects of osteoporosis. Elsevier, 2004, S. 439-447.

27. Frassetto LA, Todd KM, Morris RC Jr, Sebastian A: Worldwide incidence of hip fracture in elderly women: relation to consumption of animal and vegetable foods. Journals of Gerontology Series A-Biol. Sciences \& Med. Sciences. 2000;55(10):M585-592.

28. Zwart SR, Hargens AR, Smith SM: The ration of animal protein to potassium intake is a predictor of bone resorption in space flight analogues and in ambulatory subjects. Am J Clin Nutr 2004:80:1058-1065.

29. Spence LA, Lipscomb ER, Cadogan J et al: The effect of soy protein and soy isoflavones on calcium metabolism in postmenopausal women: a randomized crossover study. Am J Clin Nutr 2005:81:916-922.

30. Tucker KL, Hannan MT, Chen H, Cupples LA, Wilson PWF, Kiel DP. Potassium, magnesium, and fruit and vegetable intakes are associated with greater bone mineral density in elderly men and women. AM J Clin Nutr 1999;69:727-736.

31. New SA, Robins SP, Campbell MK, Martin JC, Garton MJ, Bolton-Smith C, Grubb DA, Lee SJ, Reid DM. Dietary influences on bone mass and bone metabolism: further evidence of a positive link between fruit and vegetable consumption and bone health. Am J Clin Nutr 2000:71:142-151.

32. Macdonald HM, New SA, Golden MHN et al: Nutritional associations with bone loss during the menopausal transition: evidence of a beneficial effect of calcium, alcohol, and fruit and vegetable nutrients and of a detrimental effect of fatty acids. Am J Clin Nutr 2004;79: 155-165.

33. Tylavsky FA, Holliday K, Danish $R$ et al: Fruit and vegetable intakes are an independent predictor of bone size in early pubertal children. Am J Clin Nutr 2004;7:311-317.

34. New S: Do vegetarians have a normal bone mass? Osteoporos Int 2004;15:679-88.

35. Buclin T, Cosma M, Appenzeller M, et al.: Diet acids and alkalis influence calcium retention in bone. Osteoporosis Int 2001:12:493-499.

36. Frassetto LA, Todd KM, Morris RC Jr, Sebastian A: Estimation of net endogenous noncarbonic acid production in humans from diet potassium and protein contents. Am J Clin Nutr 1998;68:576-583

37. New S, MacDonald HM, Campbell MK, et al:
Lower estimates of net endogenous non carbonic acid production are positively associated with indexes of bone health in prememopausal and perimenopausal women. Am J Clin Nutr 2004;79:131-138.

38. Maurer M, Riesen W, Muser J et al: Neutralization of western diet inhibits bone resorption independently of $K$ intake and reduces cortisol secretion in humans. Am J Physiol Renal 2003;284:32-40.

39. Chen YM, Ho SC, Lam SSH et al: Soy isoflavones have a favorable effect on bone loss in chinese postmenopausal women with lower bone mass: a double-blind, randomized, controls trial. J Clin Endocrin Metab 2003;88(10):4740-4747. 